\title{
Perspectives
}

\section{Normal reference ranges for semen quality and their relations to fecundity}

\author{
Niels E. Skakkebaek
}

University Department of Growth and Reproduction, Rigshospitalet, Copenhagen DK-2100, Denmark

\begin{abstract}
Several recent studies have shown that the fecundity of a man decreases progressively with sperm concentrations below 40 million spermatozoa per $\mathrm{mL}$. Therefore, it is unfortunate that the new World Health Organization guidelines for semen analysis recommend lowering the lower cutoff value for normal sperm concentration from 20 to 15 million spermatozoa per $\mathrm{mL}$. As a result large groups of subfertile men across the world may not receive appropriate andrological help in the future.
\end{abstract}

Asian Journal of Andrology (2010) 12: 95-98. doi: 101.1038/aja.2008.43

Keywords: semen quality, infertility, sperm morphology, fecundity

\section{Introduction}

Every day doctors all over the world are using numerous laboratory tests on blood, urine, semen and other body fluids to predict health and degree of disease. In order to distinguish between healthy and diseased individuals the so-called normal reference levels have been of enormous significance in clinical chemistry and haematology. Homeostasis keeps many such parameters remarkably stable in healthy populations and mean $\pm 2 \mathrm{SD}$ or the range between the 2.5 and 97.5 percentiles make very good sense as reference levels. However, it has also been acknowledged that there may be significant exceptions owing to geographic or temporal differences in normal reference ranges. For example, thyroid hormone levels may differ from one area to another owing to variations in intake of iodine through food and a liver parameter may change substantially over time in a given population. Recently, Lee et al. [1] reported a strong secular trend

Correspondence to: Prof. Niels E. Skakkebaek, University Department of Growth and Reproduction, Rigshospitalet, Copenhagen DK2100, Denmark.

Fax:+45-3545-6054_E-mail: nes@rh.dk

Received: 1 November 2008 Revised: 4 November 2008
(180\% increase) in serum gamma-glutamyltransferase from 1996 to 2003 in South Korean men [1]. Therefore, standards on reference levels based on results from $95 \%$ of a healthy population may not always be applicable. Instead, they may be limited to a given area and a given time. Another crucial point is that reference ranges, based on averages and a given range of a population may not always be in line with a 'desirable' normal range. This might be true particularly for industrialised societies with rapidly changing lifestyles resulting in changed nutrition, increasing body mass index and increased insulin and blood glucose levels. In our modern societies where a significant proportion of people have undiagnosed type- 2 diabetes, desirable normal fasting blood glucose levels cannot be based on simple calculation of levels obtained from $95 \%$ of the population. Likewise, reference ranges for desirable BMI levels of a society cannot be based on $95 \%$ of populations, where obesity is a growing problem.

In this commentary, I shall focus on special problems related to normal reference ranges for semen quality and relate my comments to the recently published World Health Organization (WHO) guidelines for analysis of semen [2]. Several parameters, including motility, morphology and number of spermatozoa are important. However, for simplicity I shall focus my comments on sperm counts. 


\section{2 'Normal' reference ranges versus ranges found in populations of individuals in good health}

How should a reference range for semen quality ideally be constructed? It depends very much on the expected use of the data. Several scenarios exist: 1) Health authorities may be interested in the range of sperm counts of a given population. In such cases a 95\% range of all individuals in that population, including those with known super- and sub-fertility seems to be the relevant reference dataset. 2) However, another important question is: what is the ideal standard for a fertile population? Such reference ranges should, in my opinion, depend upon their intended clinical uses. A reference range for semen quality to be used in clinical andrology to detect men who need andrology management should be split into two categories. The first is a 'grey' infertile/subfertile zone where, on the one hand it can be predicted that the ability to reproduce is preserved to some extent, particularly if the partner has optimal reproductive capacity, on the other hand a significant proportion in this group will have such poor reproductive capacity that they will seldom achieve a pregnancy. The second category reflects a normal reference range, where full reproductive capacity is present. Only a semen quality above this cut-off level can be considered normal with reasonable certainty from a biological, as well as a practical clinical viewpoint.

\section{Historical aspects on attempts to define lowest levels of semen parameters usually not associated with infertility}

A pivotal question therefore remains: what are the lowest ranges of semen parameters compatible with good fertility, above which men should normally not be referred for further clinical and laboratory investigations in the first phase of an investigation of an infertile couple? In his thesis from 1944 [3] the pioneering Danish andrologist Richard Hammen wrote that a normally fertile semen sample should fulfil certain criteria, including a concentration of 60 million spermatozoa per $\mathrm{mL}$ or more. He was in agreement with John McLeod in New York, who in 1946 [4] wrote 'I think that if we are to select a count level to represent the demarcation line between 'poor and fair' fertility that of 60 million spermatozoa per $\mathrm{mL}$ would be a wise choice'. How did these investigators in andrology reach that conclusion? Was it just their personal view or did they have data? Yes indeed, there were data, although perhaps not as stringently reported as those of the recently revised WHO manual. McLeod [5] had undertaken an investigation of 100 young medical students and found that the vast majority had sperm counts above 60 million spermatozoa per $\mathrm{mL} ; 65 \%$ had in fact more than 100 million spermatozoa per $\mathrm{mL}$ [5]. He also, like Hammen, had vast experience of semen analysis of men in barren couples, including men in couples where the partner had an infertility problem. However, later, McLeod changed his view and suggested that a concentration of 20 million spermatozoa per $\mathrm{mL}$ should be the lower limit for normal sperm concentration [6].

\section{Reference values of the recently published WHO manual for semen analysis}

A lower cut-off value of 20 million spermatozoa per $\mathrm{mL}$ has also been part of the WHO guidelines for semen analysis since the first edition of the manual was published in 1980. However, the most recent version of the WHO guidelines, just published [2], has lowered the cut-off value to 15 million spermatozoa per $\mathrm{mL}$. This changed version is based on excellent statistical methods used to analyse data from large international studies on semen quality of men involved in recent conceptions. The international committee behind the publication should be complemented for the statistical quality of the data included and the elegant presentation of them in this new publication. The committee's recommendations will be useful in some settings. Epidemiologists interested in semen quality of populations of fertile men will probably find the data useful as a modern reference to semen among fathers of recently born children.

However, I have some concerns. The most important problem is that the new WHO guidelines may be less useful for practising physicians working on infertile couples. In our daily work we need a demarcation line between semen quality with 'full reproductive competence' and that of subfertile men who should be referred for andrological work-up. Several recent publications show that a sperm concentration of 15 million spermatozoa per $\mathrm{mL}$ is far too low in general to be associated with normal fertility, although some will be able to achieve a conception. Even the previous WHO cut-off value of 20 million spermatozoa per $\mathrm{mL}$ was probably too low to identify a significant group of males who need to be referred to andrology experts [7]. We have suggested a higher cut-off value of 40 million spermatozoa per $\mathrm{mL}$, on the basis of a prospective study of first time pregnancy planners (Figure 1) [8]. Similarly, Slama [9], Guzick [10] and Jedrzejczak [11] have recently published extensive studies suggesting that even values as high as 50-60 million spermatozoa per $\mathrm{mL}$ should be used as the lower cut-off level for full reproductive competence.

Another, although minor, concern about the database behind the new version is that extramarital relationships sometimes result in misattributed paternity. This is a general problem in studies involving semen quality, but 
the chance that the biological origin may not be the man who delivered the semen sample may perhaps be higher in cases where the partner has poor semen quality. This fact could be responsible for some of the cases associating a man with an extremely low sperm count with a pregnancy.

Another, often neglected, consequence of inappropriate reference values for normal semen quality is the spin-off to medical science where normal controls are used for comparison with patients. In the andrological literature from the recent couple of decades there are numerous examples where men with sperm concentrations $>20$ million spermatozoa per $\mathrm{mL}$ are classified as 'normal', although several of such patients may, in fact, be subfertile owing to reduced spermatogenesis. With a change to an even lower cut-off value of 15 million spermatozoa per $\mathrm{mL}$ as suggested in the new WHO manual, more subfertile men may be used as normal controls, making the studies less powerful or, in some cases, even meaningless.

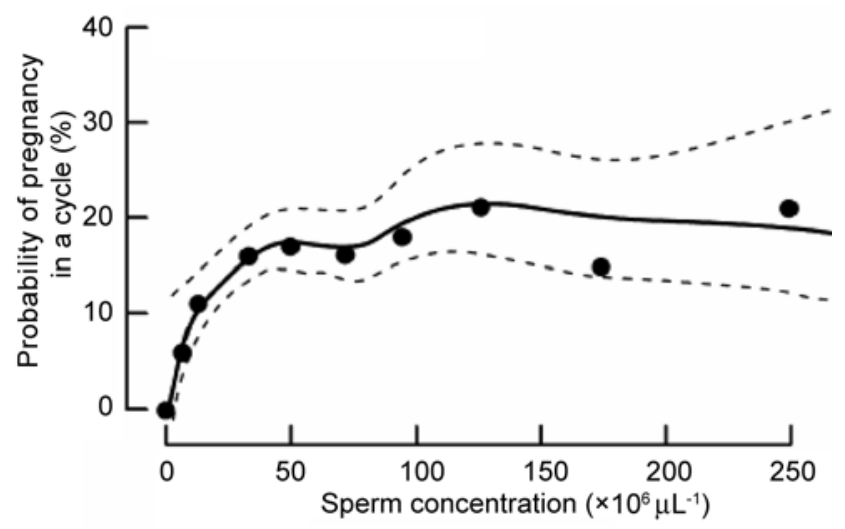

Figure 1. From Bonde et al. [8] (with permission): 430 couples with no earlier reproductive experience, 20-35 years of age, participated in a study on the association between semen quality and the probability of conception in a single menstrual cycle. The couples discontinued the use of contraception and were followed up for six menstrual cycles or until a pregnancy was verified within this period. Each man was asked to provide a semen sample at enrolment. Women kept a daily record of vaginal bleeding and sexual activity. The association between semen quality and likelihood of pregnancy was assessed by logistic regression adjusted for sexual activity and female factors associated with low fertility. There were $165(65.0 \%)$ among those with a sperm concentration of 40 million per $\mathrm{mL}$ and $84(51.2 \%)$ among those with lower sperm concentrations. The probability of conception increased with increase in sperm concentration up to 40 million per $\mathrm{mL}$, but any higher sperm concentration was not associated with the additional likelihood of pregnancy.

\section{Suggestions for the future}

My suggestion is that relatively small, but important, additions should be made as soon as possible in the new version of the WHO guidelines [2]. Otherwise the guidelines may do more harm than good. Two cutoff values for sperm counts seem needed. One of them should be much higher than the proposed, probably in the range of 40 million spermatozoa per $\mathrm{mL}$, as previously suggested [12]. It is common knowledge that unless a man has azoospermia, conception may occur. Therefore it is not surprising that the $\mathrm{WHO}$ databases on semen quality of men whose partners were pregnant contained some very low values. Nevertheless, the fecundity of a man decreases progressively with sperm concentrations below 40 million per $\mathrm{mL}$. The other cut-off value could be 15 million spermatozoa per $\mathrm{mL}$ as suggested. Thus, the area between 15 and 40 million spermatozoa per $\mathrm{mL}$ would delineate a grey subfertility zone.

In addition to revising reference limits for sperm counts the committee should perhaps look again at other cut-off values for semen quality parameters, including 'normal forms'. Here the proposed new lower reference limit of $4 \%$ normal forms (using strict criteria) seems to be rather low, neglecting results from recent studies suggesting that values up to $9 \%-12 \%$ normal forms may be associated with subfertility or infertility [10].

In conclusion, a high proportion of subfertile men in involuntary childless relationships have parameters of semen quality above the new WHO proposed cut-off values. If the new guidelines are left unchanged, a large group of subfertile men all over the world may not receive appropriate andrological help in the future. In addition, reproductive biologists will be misled about how to define a man with normal spermatogenesis.

\section{References}

1 Lee DH, Ha MH, Kam S, Chun B, Lee J, et al. A strong secular trend in serum gamma-glutamyltransferase from 1996 to 2003 among South Korean men. Am J Epidemiol 2006; 163: 57-65.

2 World Health Organisation. WHO Laboratory Manual for the Examination and Processing of Human Semen, 5th ed. Geneva: World Health Organization; 2010.

3 Hammen R. Studies on impaired fertility in man with special reference to the male, 1st edn. Koebenhavn: Einar Munksgaard; 1944.

4 MacLeod J. The semen specimen. Laboratory examination. In: Engle ET, editor. Conference on Diagnosis in Sterility. Springfield: Charles C Thomas; 1946. p3-15.

5 MacLeod J, Heim LM. Characteristics and variations in semen specimens in 100 normal young men. J Urol 1945; 54: 474-82.

6 MacLeod J, Wang Y. Male fertility potential in terms of semen quality: a review of the past, a study of the present. Fertil Steril 1979; 31: 103-16. 
7 World Health Organization. WHO Laboratory Manual for the Examination of Human Semen and Sperm-Cervical Mucus Interaction, 3rd ed. Cambridge: Cambridge University Press; 1992.

8 Bonde JP, Ernst E, Jensen TK, Hjollund NH, Kolstad $\mathrm{H}$, et al. Relation between semen quality and fertility: a population-based study of 430 first-pregnancy planners. Lancet 1998; 352: 1172-7.

9 Slama R, Eustache F, Ducot B, Jensen TK, Jørgensen N, et al. Time to pregnancy and semen parameters: a cross-sectional study among fertile couples from four European cities. Hum
Reprod 2002; 17: 503-15.

10 Guzick DS, Overstreet JW, Factor-Litvak P, Brazil CK, Nakajima $\mathrm{ST}$, et al. Sperm morphology, motility, and concentration in fertile and infertile men. N Engl J Med 2001; 345: 1388-93.

11 Jedrzejczak P, Taszarek-Hauke G, Hauke J, Pawelczyk L, Duleba AJ. Prediction of spontaneous conception based on semen parameters. Int J Androl 2008; 31: 499-507.

12 Andersson AM, Jørgensen N, Main KM, Toppari J, Rajpert-De Meyts E, et al. Adverse trends in male reproductive health: we may have reached a crucial 'tipping point'. Int J Androl 2008; 31: 74-80. 